\title{
Some approximation results for generalized Kantorovich-type operators
}

\author{
Mohammad Mursaleen ${ }^{1}$, Faisal Khan ${ }^{1}$, Asif Khan ${ }^{1}$ and Adem Kiliçmann ${ }^{2 *}$
}

\author{
*Correspondence: \\ akilicman@putra.upm.edu.my \\ ${ }^{2}$ Department of Mathematics, \\ Faculty of Science, University Putra \\ Malaysia (UPM), Serdang, Selangor \\ Darul Ehsan 4300, Malaysia \\ Full list of author information is \\ available at the end of the article
}

\begin{abstract}
In this paper, we construct a new family of operators, prove some approximation results in A-statistical sense and establish some direct theorems for Kantorovich-type integral operators.

MSC: Primary 41A10; 41A25; 41A36

Keywords: Lagrange polynomial; Korovkin approximation theorems; A-statistical convergence; Kantorovich-type operators; positive linear operators; modulus of continuity; Peetre's K-functional; Lipschitz class
\end{abstract}

\section{Introduction and preliminaries}

Statistical convergence [1] and its variants, extensions and generalizations have been proved to be an active area of recent research in summability theory, e.g., lacunary statistical convergence [2], $\lambda$-statistical convergence [3], $A$-statistical convergence [4], statistical $A$-summability [5], statistical summability $(C, 1)[6]$, statistical summability $(H, 1)$ [7], statistical summability $(\bar{N}, p)[8]$ and statistical $\sigma$-summability [9] etc. Following the work of Gadjiv and Orhan [10], these statistical summability methods have been used in establishing many approximation theorems (e.g., $[5,11-20]$ and [21]). Recently, the statistical approximation properties have also been investigated for several operators. For instance, in [22] Butzer and Hahn operators; in [23] and [24] q-analogue of Stancu-Beta operators; in [25] Bleimann, Butzer and Hahn operators; in [26] Baskakov-Kantorovich operators; in [27] Szász-Mirakjan operators; in [28] analogues of Bernstein-Kantorovich operators; and in [29] $q$-Lagrange polynomials were defined and their statistical approximation properties were investigated. Most recently, the statistical summability of Walsh-Fourier series has been discussed in [30]. In this paper, we construct a new family of operators with the help of Erkuş-Srivastava polynomials, establish some $A$-statistical approximation properties and direct theorems.

Let us recall the following definitions.

Let $\mathbb{N}$ denote the set of all natural numbers. Let $K \subseteq \mathbb{N}$ and $K_{n}=\{k \leq n: k \in K\}$. Then the natural density of $K$ is defined by $\delta(K)=\lim _{n} n^{-1}\left|K_{n}\right|$ if the limit exists, where $\left|K_{n}\right|$ denotes the cardinality of the set $K_{n}$. A sequence $x=\left(x_{k}\right)$ of real numbers is said to be statistically convergent to $L$ (cf. Fast [1]) provided that for every $\epsilon>0$ the set $\left\{k \in \mathbb{N}:\left|x_{k}-L\right| \geq \epsilon\right\}$ has natural density zero, i.e., for each $\epsilon>0$,

$$
\lim _{n} \frac{1}{n}\left|\left\{k \leq n:\left|x_{k}-L\right| \geq \epsilon\right\}\right|=0 .
$$

O2013 Mursaleen et al.; licensee Springer. This is an Open Access article distributed under the terms of the Creative Commons Attribution License (http://creativecommons.org/licenses/by/2.0), which permits unrestricted use, distribution, and reproduction in any medium, provided the original work is properly cited. 
In this case, we write $s t-\lim _{k} x_{k}=L$. Note that every convergent sequence is statistically convergent but not conversely.

Let $A=\left(a_{n k}\right), n, k=1,2,3, \ldots$, be an infinite matrix. For a given sequence $x=\left(x_{k}\right)$, the $A$-transform of $x$ is defined by $A x=\left((A x)_{n}\right)$, where $(A x)_{n}=\sum_{k=1}^{\infty} a_{n k} x_{k}$, provided the series converges for each $n$. We say that $A$ is regular if $\lim _{n}(A x)_{n}=L=\lim x$. Let $A$ be a regular matrix.

We say that a sequence $x=\left(x_{k}\right)$ is $A$-statistically convergent to a number $L$ (cf. Kolk [4]) if for every $\epsilon>0$,

$$
\lim _{n} \sum_{k:\left|x_{k}-L\right| \geq \epsilon} a_{n k}=0
$$

In this case, we denote this limit by $s t_{A}-\lim _{n} x_{n}=L$.

Note that for $A=C_{1}:=\left(c_{j n}\right)$, the Cesàro matrix of order $1, A$-statistical convergence reduces to the statistical convergence.

\section{Construction of a new operator and its properties}

The well-known (two-variable) polynomials $g_{n}^{(\alpha, \beta)}(x, y)$, which are generated by

$$
(1-x z)^{-\alpha}(1-y z)^{-\beta}=\sum_{n=0}^{\infty} g_{n}^{(\alpha, \beta)}(x, y) z^{n} \quad\left(|z|<\min \left\{|x|^{-1},|y|^{-1}\right\}\right)
$$

are the Lagrange polynomials which occur in certain problems in statistics [31]. Recently, Chan [32] introduced and systematically investigated the multivariable extension of the classical Lagrange polynomials $g_{n}^{(\alpha, \beta)}(x, y)$. These multivariable Lagrange polynomials, which are popularly known in the literature as the Chan-Chyan-Srivastava polynomials, are generated by (see [32] and [33])

$$
\begin{aligned}
& \prod_{j=1}^{r}\left\{\left(1-x_{j} z\right)^{-\alpha_{j}}\right\}=\sum_{n=0}^{\infty} g_{n}^{\left(\alpha_{1}, \ldots, \alpha_{r}\right)}\left(x_{1}, \ldots, x_{r}\right) z^{n}, \\
& \quad \alpha_{j} \in \mathbb{C}(j=1,2, \ldots, r) ;|z|<\min \left\{\left|x_{1}\right|^{-1}, \ldots,\left|x_{r}\right|^{-1}\right\} .
\end{aligned}
$$

Clearly, the defined generating function (2.2) yields the explicit representation given by [34, p.140, Eq. (6)]

$$
g_{n}^{\left(\alpha_{1}, \ldots, \alpha_{r}\right)}\left(x_{1}, \ldots, x_{r}\right)=\sum_{k_{1}+\cdots+k_{r}=n}\left(\alpha_{1}\right)_{k_{1}} \cdots\left(\alpha_{r}\right)_{k_{r}} \frac{x_{1}^{k_{1}}}{! k_{1}} \cdots \frac{x_{r}^{k_{r}}}{! k_{r}}
$$

or, equivalently, by [14, p.522, Eq. (17)]

$$
\begin{aligned}
& g_{n}^{\left(\alpha_{1}, \ldots, \alpha_{r}\right)}\left(x_{1}, \ldots, x_{r}\right) \\
& \quad=\sum_{n_{r-1}=0}^{n} \sum_{n_{r-2}=0}^{n_{r-1}} \cdots \sum_{n_{1}=0}^{n_{2}} \frac{\left(\alpha_{1}\right)_{n_{1}}\left(\alpha_{2}\right)_{n_{2}-n_{1}} \cdots\left(\alpha_{r}\right)_{n-n_{r}-1}}{n_{1} !\left(n_{2}-n_{1}\right) ! \cdots\left(n-n_{r-1}\right) !} x_{1}^{n_{1}} x_{2}^{n_{2}-n_{1}} \cdots x_{r}^{n-n_{r-1}} .
\end{aligned}
$$


On the other hand, Altin and Erkuş [34] presented a multivariable extension of the socalled Lagrange-Hermite polynomials generated by

$$
\begin{aligned}
& \prod_{j=1}^{r}\left\{\left(1-x_{j} z^{j}\right)^{-\alpha_{j}}\right\}=\sum_{n=0}^{\infty} h_{n}^{\left(\alpha_{1}, \ldots, \alpha_{r}\right)}\left(x_{1}, \ldots, x_{r}\right) z^{n}, \\
& \alpha_{j} \in \mathbb{C}(j=1,2, \ldots, r) ;|z|<\min \left\{\left|x_{1}\right|^{-1}, \ldots,\left|x_{r}\right|^{-1 / r}\right\} .
\end{aligned}
$$

The case $r=2$ of the polynomials given by (2.5) corresponds to the familiar (two-variable) Lagrange-Hermite polynomials considered by Dattoli et al. [23].

The multivariable polynomials

$$
U_{n, \ell_{1}, \ldots, \ell_{r}}^{\left(\alpha_{1}, \ldots, \alpha_{r}\right)}\left(x_{1}, \ldots, x_{r}\right)
$$

which are defined by the following generating function [32, p.268, Eq. (3)]:

$$
\begin{aligned}
& \prod_{j=1}^{r}\left\{\left(1-x_{j} z^{\ell_{j}}\right)^{-\alpha_{j}}\right\}=\sum_{n=0}^{\infty} U_{n, \ell_{1}, \ldots, \ell_{r}}^{\left(\alpha_{1}, \ldots, \alpha_{r}\right)}\left(x_{1}, \ldots, x_{r}\right) z^{n}, \\
& \quad \alpha_{j} \in \mathbb{C}(j=1,2, \ldots, r) ; \ell_{j} \in \mathbb{N}(j=1,2, \ldots, r) ;|z|<\min \left\{\left|x_{1}\right|^{-1 / \ell_{1}}, \ldots,\left|x_{r}\right|^{-1 / \ell_{r}}\right\},
\end{aligned}
$$

are a unification (and generalization) of several known families of multivariable polynomials including (for example) Chan-Chyan-Srivastava polynomials

$$
g_{n}^{\left(\alpha_{1}, \ldots, \alpha_{r}\right)}\left(x_{1}, \ldots, x_{r}\right)
$$

defined by (2.2) (see [35] for details). Obviously, the Chan-Chyan-Srivastava polynomials

$$
g_{n}^{\left(\alpha_{1}, \ldots, \alpha_{r}\right)}\left(x_{1}, \ldots, x_{r}\right)
$$

follow as a special case of the polynomials due to Erkuş and Srivastava [35]

$$
U_{n, \ell_{1}, \ldots, \ell_{r}}^{\alpha_{1}, \ldots, \alpha_{r}}\left(x_{1}, \ldots, x_{r}\right)
$$

when

$$
\ell_{j}=1 \quad(j=1, \ldots, r)
$$

where (as well as in what follows)

$$
\mathbb{N}=\{1,2,3, \ldots\} \quad \text { and } \quad \mathbb{N}_{0}=\{0,1,2, \ldots\}=\mathbb{N} \cup\{0\}
$$

Moreover, the Lagrange-Hermite polynomials

$$
h_{n}^{\left(\alpha_{1}, \ldots, \alpha_{r}\right)}\left(x_{1}, \ldots, x_{r}\right)
$$


follow as a special case of the polynomials [35]

$$
U_{n, \ell_{1}, \ldots, \ell_{r}}^{\left(\alpha_{1}, \ldots, \alpha_{r}\right)}\left(x_{1}, \ldots, x_{r}\right)
$$

when

$$
\ell_{j}=1 \quad(j=1, \ldots, r) .
$$

The generating function (2.6) yields the following explicit representation ([35, p.268, Eq. (4)]):

$$
U_{n, \ell_{1}, \ldots, \ell_{r}}^{\left(\alpha_{1}, \ldots, \alpha_{r}\right)}\left(x_{1}, \ldots, x_{r}\right)=\sum_{\ell_{1} k_{1}+\cdots+\ell_{r} k_{r}=n}\left(\alpha_{1}\right)_{k_{1}} \cdots\left(\alpha_{r}\right)_{k_{r}} \frac{x_{1}^{k_{1}}}{k_{1} !} \cdots \frac{x_{r}^{k_{r}}}{k_{r} !}
$$

which, in the special case when

$$
\ell_{j}=1 \quad(j=1, \ldots, r)
$$

corresponds to (2.3).

The following relationship is established between the polynomials due to Erkuş and Srivastava [35] and the Chan-Chyan-Srivastava polynomials by applying the generating functions (2.2) and (2.6) in [36].

$$
\begin{aligned}
& \sum_{n=0}^{\infty} U_{n, \ell_{1}, \ldots, \ell_{r}}^{\left(\alpha_{1}, \ldots, \alpha_{r}\right)}\left(x_{1}, \ldots, x_{r}\right) z^{n} \\
& =\prod_{i=1}^{r}\left\{\left(1-x_{i} z^{\ell_{i}}\right)^{-\alpha_{i}}\right\}=\prod_{i=1}^{r} \prod_{j=1}^{\ell_{i}}\left\{\left(1-\omega^{(i, j)} z\right)^{-\alpha_{i}}\right\} \\
& \quad=\sum_{n=0}^{\infty} g_{n}^{\left(\alpha_{1}, \ldots, \alpha_{1}, \ldots, \alpha_{r}, \ldots, \alpha_{r}\right)}\left(\omega_{11}, \ldots, \omega_{1 \ell_{1}}, \ldots, \omega_{r 1}, \ldots, \omega_{r \ell_{r}}\right) z^{n},
\end{aligned}
$$

where it is tacitly assumed that the following set:

$$
\omega^{(i, j)}: 1 \leq i \leq r \quad \text { and } \quad 1 \leq j \leq \ell_{i} \quad\left(\ell_{i} \in \mathbb{N} ; i=1, \ldots, r\right)
$$

which depends upon the $\ell_{i}$ distinct values of the factor $x_{i}^{\frac{1}{\ell_{i}}}$ occurring in the expression

$$
1-\left(x_{i}^{\frac{1}{\ell_{i}}} z\right)^{\ell_{i}} \quad(i=1, \ldots, r)
$$

exists such that

$$
\left(1-x_{i} z^{\ell_{i}}\right)^{-\alpha_{i}}=\prod_{j=1}^{\ell_{i}}\left\{\left(1-\omega^{(i, j)} z\right)^{-\ell_{j}}\right\} \quad(i=1, \ldots, r) .
$$

Thus, by assertion (2.8), we obtain the desired relationship as follows:

$$
U_{n, \ell_{1}, \ldots, \ell_{r}}^{\left(\alpha_{1}, \ldots, \alpha_{r}\right)}\left(x_{1}, \ldots, x_{r}\right)=g_{n}^{\left(\alpha_{1}, \ldots, \alpha_{1}, \ldots, \alpha_{r}, \ldots, \alpha_{r}\right)}\left(\omega^{(1,1)}, \ldots, \omega^{\left(1, \ell_{1}\right)}, \ldots, \omega^{(r, 1)}, \ldots, \omega^{\left(r, \ell_{r}\right)}\right) .
$$


Now by using the Erkuş-Srivastava multivariable polynomials given by (2.2), we introduce the following family of positive linear operators on $C[0,1]$ :

$$
\begin{aligned}
T_{n}^{\omega^{(1,1)}, \ldots, \omega^{\left(1, \ell_{1}\right)}, \ldots, \omega^{(r, 1)}, \ldots, \omega^{\left(r, \ell_{r}\right)}}(f ; x) \\
=\prod_{i=1}^{r}\left\{\left(1-x_{i} z^{\ell_{i}}\right)\right\}^{n} \sum_{m=0}^{\infty} U_{m, \ell_{1}, \ldots, \ell_{r}}^{\left(\alpha_{1}, \ldots, \alpha_{r}\right)}\left(x_{1}, \ldots, x_{r}\right) z^{m} \int_{\frac{k_{r}}{n+k_{r}-1}}^{\frac{k_{r}+1}{n+k_{r}-1}} f(t) d t \\
=\prod_{i=1}^{r} \prod_{j=1}^{\ell_{i}}\left\{\left(1-\omega^{(i, j)} z\right)\right\}^{n} \sum_{m=0}^{\infty} g_{m}\left(\omega^{(1,1)}, \ldots, \omega^{\left(1, \ell_{1}\right)}, \ldots, \omega^{(r, 1)}, \ldots, \omega^{\left(r, \ell_{r}\right)}\right) z^{n} \\
\quad \times\left(n+k_{r}-1\right) \int_{\frac{k_{r}}{n+k_{r}-1}}^{\frac{k_{r}+1}{n+k_{r}-1}} f(t) d t,
\end{aligned}
$$

where

$$
\alpha_{j} \in \mathbb{C}(j=1,2, \ldots, r) ; \quad \ell_{j} \in \mathbb{N}(j=1,2, \ldots, r) ; \quad|z|<\min \left\{\left|x_{1}\right|^{-1 / \ell_{1}}, \ldots,\left|x_{r}\right|^{-1 / \ell_{r}}\right\}
$$

Throughout this paper, we assume that

$$
\omega^{(i, j)}=\left\{\omega_{(n)}^{(i, j)}\right\}_{n \in \mathbb{N}^{\prime}}, \quad 1 \leq i \leq r \text { and } 1 \leq j \leq \ell_{i}\left(\ell_{i} \in \mathbb{N} ; i=1, \ldots, r\right),
$$

are sequences of real numbers such that

$$
0<\omega^{(i, j)}<1 .
$$

For convenience, taking $r=1, \ell_{i}=2, \alpha_{1}=\alpha_{2}=n$ in (2.9), we have

$$
\begin{aligned}
& T_{n}^{\omega^{(1,1)}, \omega^{(1,2)}}(f ; x) \\
& \quad=\left(1-\omega^{(1,1)} x\right)^{n}\left(1-\omega^{(1,2)} x\right)^{n} \sum_{m=0}^{\infty} g_{m}^{(n, n)}\left(\omega^{(1,1)}, \omega^{(1,2)}\right) x^{m} \int_{\frac{k}{n+k-1}}^{\frac{k+1}{n+k-1}} f(t) d t \\
& \quad=\left(1-\omega^{(1,1)} x\right)^{n}\left(1-\omega^{(1,2)} x\right)^{n} \sum_{m=0}^{\infty}\left\{\sum_{k_{1}=m} \frac{\left(\omega_{n}^{(1,1)}\right)^{k_{1}}}{k_{1} !}(n)_{k_{1}} \int_{\frac{k}{n+k-1}}^{\frac{k+1}{n+k-1}} f(t) d t\right\} x^{m} .
\end{aligned}
$$

Lemma 2.1 For each $x \in[0,1]$ and $n \in \mathbb{N}$,

$$
T_{n}^{\omega^{(1,1)}, \omega^{(1,2)}}\left(f_{0} ; x\right)=1 \quad\left(f_{0}(x)=1\right) .
$$

Lemma 2.2 For each $x \in[0,1]$ and $n \in \mathbb{N}$,

$$
T_{n}^{\omega^{(1,1)}, \omega^{(1,2)}}\left(f_{1} ; x\right) \leq x \omega_{n}^{(1,1)}+\frac{1}{2 n} \quad\left(f_{1}(x)=x\right) .
$$

Proof Let each $x \in[0,1]$ be fixed. Then from (2.10) we get

$$
\begin{aligned}
& T_{n}^{\omega^{(1,1)}, \omega^{(1,2)}}\left(f_{1} ; x\right) \\
& \quad=\left(1-\omega^{(1,1)} x\right)^{n}\left(1-\omega^{(1,2)} x\right)^{n} \sum_{m=0}^{\infty}\left(n+k_{1}-1\right)\left\{\sum_{k_{1}=m} \frac{\left(\omega_{n}^{(1,1)}\right)^{k_{1}}}{k_{1} !}(n)_{k_{1}} \int_{\frac{k}{n+k-1}}^{\frac{k+1}{n+k-1}} t d t\right\} x^{m}
\end{aligned}
$$




$$
\begin{aligned}
& =\left(1-\omega^{(1,1)} x\right)^{n}\left(1-\omega^{(1,2)} x\right)^{n} \sum_{m=0}^{\infty}\left(n+k_{1}-1\right)\left\{\sum_{k_{1}=m} \frac{\left(\omega_{n}^{(1,1)}\right)^{k_{1}}}{k_{1} !}(n)_{k_{1}}\left[\frac{t^{2}}{2}\right]_{\frac{k}{n+k-1}}^{\frac{k+1}{n+k-1}}\right\} x^{m} \\
& =\left(1-\omega^{(1,1)} x\right)^{n}\left(1-\omega^{(1,2)} x\right)^{n} \sum_{m=0}^{\infty} \sum_{k_{1}=m} \frac{\left(2 k_{1}+1\right)}{2(n+k-1)} \frac{\left(\omega_{n}^{(1,1)}\right)^{k_{1}}}{k_{1} !}(n)_{k_{1}} x^{m} \\
& =x \omega_{n}^{(1,1)} x\left(1-\omega^{(1,1)} x\right)^{n}\left(1-\omega^{(1,2)} x\right)^{n} \sum_{m=1}^{\infty} \sum_{k_{1}=1}^{m} \frac{\left(\omega_{n}^{(1,1)}\right)^{k_{1}-1}}{k_{1}-1 !}(n)_{k_{1}-1} x^{m-1} \\
& =\left(1-\omega^{(1,1)} x\right)^{n}\left(1-\omega^{(1,2)} x\right)^{n} \sum_{m=0}^{\infty} \sum_{k_{1}=m} \frac{1}{2(n+k-1)} \frac{\left(\omega_{n}^{(1,1)}\right)^{k_{1}}}{k_{1} !}(n)_{k_{1}} x^{m} \\
& \leq \omega_{n}^{(1,1)} x+\frac{1}{2 n}, \quad 0<\omega_{n}^{(1,1)}<1, \omega_{n}^{(1,1)} \rightarrow 1 .
\end{aligned}
$$

Lemma 2.3 For each $x \in[0,1]$ and $n \in \mathbb{N}$,

$$
T_{n}^{\omega^{(1,1)}, \omega^{(1,2)}}\left(f_{2} ; x\right) \leq x^{2}\left(\omega_{n}^{(1,1)}\right)^{2}+\frac{2 x\left(\omega_{n}^{(1,1)}\right)}{n}+\frac{1}{3 n^{2}} \quad\left(f_{2}(x)=x^{2}\right) .
$$

Proof Let each $x \in[0,1]$ be fixed. Then from (2.10) we get

$$
\begin{aligned}
& T_{n}^{\omega^{(1,1)}, \omega^{(1,2)}}\left(f_{1} ; x\right) \\
&=\left(1-\omega^{(1,1)} x\right)^{n}\left(1-\omega^{(1,2)} x\right)^{n} \sum_{m=0}^{\infty}\left(n+k_{1}-1\right)\left\{\sum_{k_{1}=m} \frac{\left(\omega_{n}^{(1,1)}\right)^{k_{1}}}{k_{1} !}(n)_{k_{1}} \int_{\frac{k}{n+k-1}}^{\frac{k+1}{n+k-1}} t^{2} d t\right\} x^{m} \\
&=\left(1-\omega^{(1,1)} x\right)^{n}\left(1-\omega^{(1,2)} x\right)^{n} \sum_{m=0}^{\infty}\left(n+k_{1}-1\right)\left\{\sum_{k_{1}=m} \frac{\left(\omega_{n}^{(1,1)}\right)^{k_{1}}}{k_{1} !}(n)_{k_{1}}\left[\frac{t^{3}}{3}\right]_{\frac{k}{n+k-1}}^{\frac{k+1}{n+k-1}}\right\} x^{m} \\
&=\left(1-\omega^{(1,1)} x\right)^{n}\left(1-\omega^{(1,2)} x\right)^{n} \sum_{m=0}^{\infty} \sum_{k=m}(n+k-1) \frac{\left(\omega_{n}^{(1,1)}\right)^{k_{1}}}{\left(k_{1}\right) !}(n)_{k_{1}} \\
& \times\left\{\frac{k_{1}^{2}}{\left(n+k_{1}-1\right)^{3}}+\frac{k_{1}}{\left(n+k_{1}-1\right)^{3}}+\frac{1}{3\left(n+k_{1}-1\right)^{3}}\right\} x^{m} \\
&= x \omega_{n}^{(1,1)}\left(1-\omega^{(1,1)} x\right)^{n}\left(1-\omega^{(1,2)} x\right)^{n} \sum_{m=1}^{\infty} \sum_{k=1}^{m}\left(\frac{k}{n+k-1}\right)\left\{\frac{\left(\omega_{n}^{(1,1)}\right)^{k_{1}-1}}{\left(k_{1}-1\right) !}(n)_{k_{1}-1}\right\} x^{m-1} \\
&+x \omega_{n}^{(1,1)}\left(1-\omega^{(1,1)} x\right)^{n}\left(1-\omega^{(1,2)} x\right)^{n} \sum_{m=1}^{\infty} \sum_{k=1}^{m}\left(\frac{1}{n+k-1}\right) \\
& \times\left\{\frac{\left(\omega_{n}^{(1,1)}\right)^{k_{1}-1}}{\left(k_{1}-1\right) !}(n)_{k_{1}-1}\right\} x^{m-1} \\
&+x \omega_{n}^{(1,1)}\left(1-\omega^{(1,1)} x\right)^{n}\left(1-\omega^{(1,2)} x\right)^{n} \sum_{m=1}^{\infty} \sum_{k=1}^{m}\left(\frac{1}{3(n+k-1)^{2}}\right)\left\{\frac{\left(\omega_{n}^{(1,1)}\right)^{k_{1}}}{k_{1} !}(n)_{k_{1}}\right\} x^{m} \\
& \leq x^{2}\left(\omega_{n}^{(1,1)}\right)^{2}\left(1-\omega^{(1,1)} x\right)^{n}\left(1-\omega^{(1,2)} x\right)^{n} \sum_{m=2}^{\infty} \sum_{k=2}^{m}\left(\frac{(n+k-2)}{n+k-1}\right) \\
& \times\left\{\frac{\left(\omega_{n}^{(1,1)}\right)^{k_{1}-2}}{\left(k_{1}-2\right) !}(n)_{k_{1}-2}\right\} x^{m-2}+\frac{2 x \omega_{n}^{(1,1)}}{n}+\frac{1}{3 n^{2}} \\
& \\
&
\end{aligned}
$$




$$
\begin{gathered}
\leq x^{2}\left(\omega_{n}^{(1,1)}\right)^{2}+\frac{2 x \omega_{n}^{(1,1)}}{n}+\frac{1}{3 n^{2}}, \\
T_{n}^{\omega^{(1,1)}, \omega^{(1,2)}}\left(f_{2} ; x\right)-f_{2}(x) \leq x^{2}\left(\left(\omega_{n}^{(1,1)}\right)^{2}-1\right)+\frac{2 x \omega_{n}^{(1,1)}}{n}+\frac{1}{3 n^{2}} .
\end{gathered}
$$

On the other hand, since

$$
0 \leq T_{n}^{\omega^{(1,1)}, \omega^{(1,2)}}\left((y-x)^{2} ; x\right)=T_{n}^{\omega^{(1,1)}, \omega^{(1,2)}}\left(f_{2} ; x\right)-2 x T_{n}^{\omega^{(1,1)}, \omega^{(1,2)}}\left(f_{1} ; x\right)+x^{2},
$$

it follows from Lemma 2.1 and Lemma 2.2 that

$$
T_{n}^{\omega^{(1,1)}, \omega^{(1,2)}}\left(f_{2} ; x\right)-f_{2}(x) \geq 2 x^{2}\left(\left(\omega_{n}^{(1,1)}\right)^{2}-1\right) .
$$

Combining (2.11) and (2.12), we have

$$
\left|T_{n}^{\omega^{(1,1)}, \omega^{(1,2)}}\left(f_{2} ; x\right)-f_{2}(x)\right| \leq x^{2}\left(1-\left(\omega_{n}^{(1,1)}\right)^{2}\right)+\frac{2 x \omega_{n}^{(1,1)}}{n}+\frac{1}{3 n^{2}} .
$$

Then, taking supremum over $x \in[0,1]$, we have

$$
\left\|T_{n}^{\omega^{(1,1)}, \omega^{(1,2)}}\left(f_{2} ; x\right)-f_{2}(x)\right\| \leq x^{2}\left(1-\left(\omega_{n}^{(1,1)}\right)^{2}\right)+\frac{2 x \omega_{n}^{(1,1)}}{n}+\frac{1}{3 n^{2}} .
$$

\section{Remark 2.1}

$$
\begin{aligned}
T_{n}^{\omega^{(1,1)}, \omega^{(1,2)}}(t-x ; x) & =x \omega_{n}^{(1,1)}-x+\frac{1}{2 n} \\
& =x\left(\omega_{n}^{(1,1)}-1\right) \frac{1}{2 n} .
\end{aligned}
$$

Remark 2.2 Let $x \in[0,1]$, since $T_{n}^{\omega^{(1,1)}, \omega^{(1,2)}}$ is linear, we get

$$
\begin{aligned}
& T_{n}^{\omega^{(1,1)}, \omega^{(1,2)}}\left((y-x)^{2} ; x\right) \\
& \quad=T_{n}^{\omega^{(1,1)}, \omega^{(1,2)}}\left(f_{2} ; x\right)-2 x T_{n}^{\omega^{(1,1)}, \omega^{(1,2)}}\left(f_{1} ; x\right)+x^{2} T_{n}^{\omega^{(1,1)}, \omega^{(1,2)}}\left(f_{0} ; x\right) \\
& \quad \leq x^{2}\left(\omega^{(1,1)}\right)^{2}+\frac{2 x \omega^{(1,1)}}{n}-2 x^{2} \omega^{(1,1)}+x^{2}-2 x\left[x \omega_{n}^{(1,1)}+\frac{1}{2 n}\right]+x^{2} \\
& \quad \leq x^{2}\left(\omega^{(1,1)}\right)^{2}+\frac{2 x \omega^{(1,1)}}{n}-2 x^{2} \omega^{(1,1)}+x^{2}+\frac{1}{3 n^{2}}-\frac{x}{n} .
\end{aligned}
$$

\section{A-statistical approximation}

Let $C[a, b]$ be a linear space of all real-valued continuous functions $f$ on $[a, b]$, and let $T$ be a linear operator which maps $C[a, b]$ into itself. We say that $T$ is positive if for every non-negative $f \in C[a, b]$, we have $T(f, x) \geq 0$ for all $x \in[a, b]$. We know that $C[a, b]$ is a Banach space with the norm

$$
\|f\|_{C[a, b]}:=\sup _{x \in[a, b]}|f(x)|, \quad f \in C[a, b] .
$$

For typographical convenience, we will write $\|\cdot\|$ in place of $\|\cdot\|_{C[a, b]}$ if no confusion arises. 
Theorem 3.1 Let $A=\left(a_{j n}\right)$ be a non-negative regular summability matrix. Then

$$
s t_{A}-\lim _{n} \omega_{n}^{(1,1)}=1
$$

if and only if for all $f \in C[0,1]$,

$$
s t_{A}-\lim _{n}\left\|T_{n}^{\omega^{(1,1)}, \omega^{(1,2)}}(f)-f\right\|=0 .
$$

Proof Suppose that (3.2) holds for all $f \in C[0,1]$. Then we have

$$
s t_{A}-\lim _{n}\left\|T_{n}^{\omega^{(1,1)}, \omega^{(1,2)}}\left(f_{1}\right)-f_{1}\right\|=0
$$

since $f_{1} \in C[0,1]$. By Lemma 2.2, we have

$$
\left\|T_{n}^{\omega^{(1,1)}, \omega^{(1,2)}}\left(f_{1}\right)-f_{1}\right\|=1-\omega_{n}^{(1,1)} .
$$

By (3.3) and (3.4), we immediately get

$$
s t_{A}-\lim _{n} \omega_{n}^{(1,1)}=1 .
$$

Conversely, suppose that (3.1) holds. Then from Lemma 2.1 we have $\lim _{n} \| T_{n}^{\omega^{(1,1)}, \omega^{(1,2)}}\left(f_{0}\right)-$ $f_{0} \|=0$. Hence

$$
s t_{A}-\lim _{n}\left\|T_{n}^{\omega^{(1,1)}, \omega^{(1,2)}}\left(f_{0}\right)-f_{0}\right\|=0 \quad\left(f_{0}(x)=1\right) .
$$

Also from Lemma 2.2 it follows that

$$
\left\|T_{n}^{\omega^{(1,1)}, \omega^{(1,2)}}\left(f_{1}\right)-f_{1}\right\|=1-\omega_{n}^{(1,1)} .
$$

Therefore, by using (3.1), we get

$$
s t_{A}-\lim _{n}\left\|T_{n}^{\omega^{(1,1)}, \omega^{(1,2)}}\left(f_{1}\right)-f_{1}\right\|=0 \quad\left(f_{1}(x):=x\right) .
$$

Now we claim that

$$
s t_{A}-\lim _{n}\left\|T_{n}^{\omega^{(1,1)}, \omega^{(1,2)}}\left(f_{2}\right)-f_{2}\right\|=0 \quad\left(f_{2}(x):=x^{2}\right) .
$$

By Lemma 2.3, we have

$$
\left\|T_{n}^{\omega^{(1,1)}, \omega^{(1,2)}}\left(f_{2}\right)-f_{2}\right\| \leq 2\left(1-\omega_{n}^{(1,1)}\right)+\frac{2 \omega_{n}^{(1,1)}}{n}+\frac{1}{3 n^{2}} .
$$

Now, for a given $\epsilon>0$, we define the following sets:

$$
\begin{aligned}
& D:=\left\{n:\left\|T_{n}^{\omega^{(1,1)}, \omega^{(1,2)}}\left(f_{2}\right)-f_{2}\right\| \geq \epsilon\right\}, \\
& D_{1}:=\left\{n: 1-\omega_{n}^{(1,1)} \geq \frac{\epsilon}{4}\right\},
\end{aligned}
$$




$$
\begin{aligned}
& D_{2}:=\left\{n: \frac{\omega^{(1,1)} n}{n} \geq \frac{\epsilon}{2}\right\}, \\
& D_{3}:=\left\{n: \frac{1}{n^{2}} \geq \epsilon\right\} .
\end{aligned}
$$

From (3.8), it is easy to see that $D \subseteq D_{1} \cup D_{2} \cup D_{3}$. Then, for each $j \in \mathbb{N}$, we get

$$
\sum_{n \in D} a_{j n} \leq \sum_{n \in D_{1}} a_{j n}+\sum_{n \in D_{2}} a_{j n}+\sum_{n \in D_{3}} a_{j n}
$$

Using (3.3), we get

$$
s t_{A}-\lim _{n}\left(1-\omega_{n}^{(1,1)}\right)=0
$$

and

$$
s t_{A}-\lim _{n} \frac{\omega_{n}^{(1,1)}}{n}=0 .
$$

Now, using the above facts and taking the limit as $j \rightarrow \infty$ in (3.9), we conclude that

$$
\lim _{j} \sum_{n \in D} a_{j n}=0
$$

which gives (3.7). Now, combining (3.5)-(3.7), and using the statistical version of the Korovkin approximation theorem (see Gadjiv and Orhan [10], Theorem 1), we get the desired result.

This completes the proof of the theorem.

In a similar manner, we can extend Theorem 3.1 to the $(i, j)$-dimensional case for the operators $T_{n}^{\omega^{(1,1)}, \ldots, \omega^{\left(1, \ell_{1}\right)}, \ldots, \omega^{(r, 1)}, \ldots, \omega^{(r, \ell)}}(f ; x)$ given by $(2.9)$ as follows.

Theorem 3.2 Let $A=\left(a_{j n}\right)$ be a non-negative regular summability matrix. Then

$$
s t_{A}-\lim _{n} \omega_{n}^{(i, j)}=1
$$

if and only if for all $f \in C[0,1]$,

$$
s t_{A}-\lim _{n}\left\|T_{n}^{\omega^{(1,1)}, \ldots, \omega^{\left(1, \ell \ell_{1}\right)}, \ldots, \omega^{(r, 1)}, \ldots, \omega^{(r, \ell r)}}(f)-f\right\|=0 .
$$

Remark 3.1 If in Theorem 3.2 we replace $A=\left(a_{j n}\right)$ by the identity matrix, we immediately get the following theorem which is a classical case of Theorem 3.2.

Theorem $3.3 \lim _{n} \omega_{n}^{(i, j)}=1$ if and only if for all $f \in C[0,1]$, the sequence

$$
T_{n}^{\omega^{(1,1)}, \ldots, \omega^{\left(1, \ell_{1}\right)}, \ldots, \omega^{(r, 1)}, \ldots, \omega^{(r, \ell)}}(f)
$$

is uniformly convergent to $f$ on $[0,1]$. 
Finally, we display an example which satisfies all the hypotheses of Theorem 3.2, but not of Theorem 3.3. Therefore, this indicates that our $A$-statistical approximation in Theorem 3.2 is stronger than its classical case.

Take $A=C_{1}:=\left(c_{j n}\right)$, the Cesàro matrix of order 1 and

$$
\omega^{(i, j)}:=\left(\omega_{n}^{(i, j)}\right)_{n \in \mathbb{N}} \quad(j=1, \ldots, r-1)
$$

are sequences of real numbers defined by

$$
\omega_{n}^{(i, j)}:= \begin{cases}\frac{1}{2} & \text { if } n=m^{2}(m \in \mathbb{N}) ; \\ 1-\frac{1}{n+i j} & \text { otherwise. }\end{cases}
$$

We then observe that

$$
0<\omega_{n}^{(i, j)}<1 \quad(n \in \mathbb{N})
$$

and also that

$$
s t_{A}-\lim _{n} \omega_{n}^{(i, j)}=1 .
$$

Therefore, by Theorem 3.2, we have that for all $f \in C[0,1]$,

$$
s t_{A}-\lim _{n}\left\|T_{n}^{\omega^{(1,1)}, \ldots, \omega^{\left(1, \ell_{1}\right)}, \ldots, \omega^{(r, 1)}, \ldots, \omega^{(r, \ell r)}}(f)-f\right\|=0 .
$$

However, since the sequence $\omega_{n}^{(i, j)}$ defined by (3.10) is non-convergent, Theorem 3.3 does not hold in this case.

\section{Direct theorems}

By $C_{B}[0,1]$, we denote the space of all real-valued continuous bounded functions $f$ on the interval $[0,1]$, the norm $\|\cdot\|$ on the space $C_{B}[0,1]$ is given by

$$
\|f\|=\sup _{0 \leq x \leq 1}|f(x)| .
$$

Peetre's $K$-functional is defined by

$$
K_{2}(f, \delta)=\inf \left[\left\{\|f-g\|+\delta\left\|g^{\prime \prime}\right\|: g \in W^{2}\right\}\right],
$$

where

$$
W^{2}=\left\{g \in C_{B}[0,1]: g^{\prime}, g^{\prime \prime} \in C_{B}[0,1]\right\} .
$$

By [14] there exists a positive constant $c>0$ s.t.

$$
K_{2}(f, \delta) \leq c w_{2}\left(f, \delta^{1 / 2}\right), \quad \delta>0,
$$


where the second-order modulus of smoothness is

$$
w_{2}(f, \sqrt{\delta})=\sup _{0 \leq h \leq \sqrt{\delta}} \sup _{0 \leq x \leq 1}|f(x+2 h)-2 f(x+h)+f(x)| .
$$

Also, for $f \in C_{B}[0,1]$, the usual modulus of continuity is given by

$$
w(f, \delta)=\sup _{0 \leq h \leq \delta} \sup _{0 \leq x \leq 1}|f(x+h)-f(x)|
$$

Theorem 4.1 Let $f \in C_{B}[0,1]$ and $0 \leq \omega_{n}^{(i, j)}<1$. Then, for all $x \in[0,1]$ and $n \in \mathbb{N}$, there exists an absolute constant $C>0$ s.t.

$$
\left|T_{n}^{\omega^{(1,1)}, \omega^{(1,2)}}(f ; x)-f(x)\right| \leq C w_{2}\left(f, \delta_{n}(x)\right)
$$

where

$$
\delta_{n}^{2}(x)=x^{2}\left[\left(\omega_{n}^{(1,1)}\right)^{2}-2 \omega_{n}^{(1,1)}+1\right]+\frac{x \omega_{n}^{(1,1)}}{n} .
$$

Proof Let $g \in W^{2}$. From Taylor's expansion

$$
g(t)=g(x)+g^{\prime}(x)(t-x)+\int_{x}^{t}(t-x) g^{\prime \prime} u d u, \quad t \in[0,1],
$$

and from Lemmas (2.1), (2.2) and (2.3), we get

$$
T_{n}^{\omega^{(1,1)}, \omega^{(1,2)}}(g, x)=g(x)+T_{n}^{\omega^{(1,1)}, \omega^{(1,2)}}\left(\int_{x}^{t}(t-x) g^{\prime \prime}(u) d u, x\right),
$$

hence

$$
\begin{aligned}
\left|T_{n}^{\omega^{(1,1)}, \omega^{(1,2)}}(g, x)-g(x)\right| & \leq\left|T_{n}^{\omega^{(1,1)}, \omega^{(1,2)}}\left(\int_{x}^{t}(t-x) g^{\prime \prime}(u) d u, x\right)\right| \\
& \leq\left|L_{n}^{u^{(1)}, u^{(2)}}\left((t-x)^{2}, x\right)\right|\left\|g^{\prime \prime}\right\| .
\end{aligned}
$$

Using Remark 2.2, we obtain

$$
\left|T_{n}^{\omega^{(1,1)}, \omega^{(1,2)}}(g, x)-g(x)\right| \leq\left[x^{2}\left(\omega^{(1,1)}\right)^{2}+\frac{2 x \omega^{(1,1)}}{n}-2 x^{2} \omega^{(1,1)}+x^{2}+\frac{1}{3 n^{2}}-\frac{x}{n}\right]\left\|g^{\prime \prime}\right\| .
$$

On the other hand, by the definition of $T_{n}^{\omega^{(1,1)}, \omega^{(1,2)}}(f, x)$, we have

$$
\left|T_{n}^{\omega^{(1,1)}, \omega^{(1,2)}}(f ; x)\right| \leq\|f\| .
$$

Next

$$
\begin{aligned}
& \left|T_{n}^{\omega^{(1,1)}, \omega^{(1,2)}}(f, x)-f(x)\right| \\
& \quad \leq\left|T_{n}^{\omega^{(1,1)}, \omega^{(1,2)}}((f-g) ; x)-(f-g)(x)\right|+\left|T_{n}^{\omega^{(1,1)}, \omega^{(1,2)}}(g, x)-g(x)\right| \\
& \quad \leq\|f-g\|+\left[x^{2}\left(\omega^{(1,1)}\right)^{2}+\frac{2 x \omega^{(1,1)}}{n}-2 x^{2} \omega^{(1,1)}+x^{2}+\frac{1}{3 n^{2}}-\frac{x}{n}\right]\left\|g^{\prime \prime}\right\| .
\end{aligned}
$$


Hence, taking infimum on the right-hand side over all $g \in W^{2}$, we get

$$
\left|T_{n}^{(1,1), \omega^{(1,2)}}(f, x)-f(x)\right| \leq C K_{2}\left(f, \delta_{n}^{2}(x)\right) .
$$

In view of the property of $K$-functional, for every $0<\omega_{n}^{(i, j)}<1$, we get

$$
\left|T_{n}^{(1,1), \omega^{(1,2)}}(f, x)-f(x)\right| \leq C w_{2}\left(f, \delta_{n}(x)\right)
$$

This completes the proof of the theorem.

Theorem 4.2 Let $f \in C_{B}[0,1]$ be such that $f^{\prime}, f^{\prime \prime} \in C_{B}[0,1]$ and $0<\omega_{n}^{(i, j)}<1, j=1,2,3, \ldots, n$, such that $\omega_{n}^{(i, j)} \rightarrow 1$ as $n \rightarrow \infty$. Then the following equality holds:

$$
\lim _{n \rightarrow \infty} n\left(T_{n}^{\omega^{(1,1)}, \omega^{(1,2)}}(f, x)-f(x)\right)=\frac{x}{2} f^{\prime \prime}(x)
$$

uniformly on $[0,1]$.

Proof By the Taylor's formula, we may write

$$
f(t)=f(x)+f^{\prime}(x)(t-x)+\frac{1}{2} f^{\prime \prime}(x)(t-x)^{2}+r(t, x)(t-x)^{2}
$$

where $r(t, x)$ is the remaining term and $\lim _{t \rightarrow x} r(t, x)=0$. Applying $T_{n}^{\omega^{(1,1)}, \omega^{(1,2)}}(f ; x)$ to $(4.1)$, we obtain

$$
\begin{aligned}
n & \left(T_{n}^{\omega^{(1,1)}, \omega^{(1,2)}}(f, x)-f(x)\right) \\
= & n T_{n}^{\omega^{(1,1)}, \omega^{(1,2)}}(t-x ; x) f^{\prime}(x) \\
& +n T_{n}^{\omega^{(1,1)}, \omega^{(1,2)}}\left((t-x)^{2} ; x\right) \frac{f^{\prime \prime}(x)}{2}+n T_{n}^{\omega^{(1,1)}, \omega^{(1,2)}}\left(r(t, x)(t-x)^{2} ; x\right) .
\end{aligned}
$$

By the Cauchy-Schwartz inequality, we have

$$
T_{n}^{\omega^{(1,1)}, \omega^{(1,2)}}\left(r(t, x)(t-x)^{2} ; x\right) \leq \sqrt{T_{n}^{\omega^{(1,1)}, \omega^{(1,2)}}\left(r^{2}(t, x)^{2} ; x\right)} \sqrt{T_{n}^{\omega^{(1,1)}, \omega^{(1,2)}}\left((t-x)^{4} ; x\right)} .
$$

Observe that $r^{2}(x, x)=0$ and $r^{2}(\cdot, x) \in C[0,1]$. Then it follows from Theorem 4.1 that

$$
\lim _{n \rightarrow \infty} T_{n}^{(1,1), \omega^{(1,2)}}\left(r^{2}(t, x) ; x\right)=r^{2}(x, x)=0
$$

uniformly with respect to $x \in[0,1]$.

Now, from (4.2), (4.3) and Remark 2.2, we get

$$
\lim _{n \rightarrow \infty} n T_{n}^{\omega^{(1,1)}, \omega^{(1,2)}}\left(r(t, x)(t-x)^{2} ; x\right)=0 .
$$


Finally, using Remark 2.1, we get the following:

$$
\begin{aligned}
\lim _{n \rightarrow \infty} n\left(T_{n}^{\omega^{(1,1)}, \omega^{(1,2)}}(f, x)-f(x)\right)= & \lim _{n \rightarrow \infty} n\left(f^{\prime}(x) T_{n}^{\omega^{(1,1)}, \omega^{(1,2)}}((t-x) ; x)\right) \\
& +\frac{1}{2} f^{\prime \prime}(x) T_{n}^{\omega^{(1,1)}, \omega^{(1,2)}}\left((t-x)^{2} ; x\right) \\
& +\frac{1}{2} f^{\prime \prime}(x) T_{n}^{\omega^{(1,1)}, \omega^{(1,2)}}\left((t-x)^{2} ; x\right) \\
& +T_{n}^{\omega^{(1,1)}, \omega^{(1,2)}}\left(r(t, x)(t-x)^{2} ; x\right) \\
& =\frac{x}{2} f^{\prime \prime}(x) .
\end{aligned}
$$

\section{Competing interests}

The authors declare that they have no competing interests.

\section{Authors' contributions}

The authors contributed equally and significantly in writing this paper. All authors read and approved the final manuscript.

\section{Author details}

'Department of Mathematics, Aligarh Muslim University, Aligarh, 202002, India. ${ }^{2}$ Department of Mathematics, Faculty of Science, University Putra Malaysia (UPM), Serdang, Selangor Darul Ehsan 4300, Malaysia.

Received: 25 September 2013 Accepted: 18 November 2013 Published: 17 Dec 2013

\section{References}

1. Fast, H: Sur la convergence statistique. Colloq. Math. 2, 241-244 (1951)

2. Fridy, JA, Orhan, C: Lacunary statistical convergence. Pac. J. Math. 160, 43-51 (1993)

3. Mursaleen, M, Alotaibi, A, Mohiuddine, SA: Statistical convergence through de la Vallée-Poussin mean in locally solid Riesz spaces. Adv. Differ. Equ. 2013, Article ID 66 (2013). doi:10.1186/1687-1847-2013-66

4. Kolk, E: Matrix summability of statistically convergent sequences. Analysis 13, 77-83 (1993)

5. Edely, $\mathrm{OHH}$, Mursaleen, M: On statistical A-summability. Math. Comput. Model. 49, 672-680 (2009)

6. Moricz, F: Tauberian conditions under which statistical convergence follows from statistical summability $(C, 1)$. J. Math. Anal. Appl. 275, 277-287 (2002)

7. Moricz, F: Theorems relating to statistical harmonic summability and ordinary convergence of slowly decreasing or oscillating sequences. Analysis 24, 127-145 (2004)

8. Moricz, F, Orhan, C: Tauberian conditions under which statistical convergence follows from statistical summability by weighted means. Studia Sci. Math. Hung. 41(4), 391-403 (2004)

9. Mursaleen, M, Edely, OHH: On the invariant mean and statistical convergence. Appl. Math. Lett. 22, 1700-1704 (2009)

10. Gadjiv, AD, Orhan, C: Some approximation theorems via statistical convergence. Rocky Mt. J. Math. 32, 129-138 (2002)

11. Demirci, K, Dirik, F: Approximation for periodic functions via statistical $\sigma$-convergence. Math. Commun. 16, 77-84 (2011)

12. Edely, $\mathrm{OHH}$, Mohiuddine, SA, Noman, AK: Korovkin type approximation theorems obtained through generalized statistical convergence. Appl. Math. Lett. 23, 1382-1387 (2010)

13. Mohiuddine, SA, Alotaibi, A, Mursaleen, M: Statistical summability $(C, 1)$ and a Korovkin type approximation theorem. J. Inequal. Appl. 2012, Article ID 172 (2012). doi:10.1186/1029-242X-2012-172

14. Devore, RA, Lorentz, GG, Constructive Approximation. Springer, Berlin (1993)

15. Mursaleen, M, Alotaibi, A: Statistical summability and approximation by de la Vallée-Poussin mean. Appl. Math. Lett. 24, 320-324 (2011). Erratum: Appl. Math. Lett. 25, 665 (2012)

16. Mursaleen, M, Karakaya, V, Ertürk, M, Gürsoy, F: Weighted statistical convergence and its application to Korovkin type approximation theorem. Appl. Math. Comput. 218, 9132-9137 (2012)

17. Srivastava, HM, Mursaleen, M, Khan, A: Generalized equi-statistical convergence of positive linear operators and associated approximation theorems. Math. Comput. Model. 55, 2040-2051 (2012)

18. Belen, C, Mursaleen, M, Yildirim, M: Statistical A-summability of double sequences and a Korovkin type approximation theorem. Bull. Korean Math. Soc. 49(4), 851-861 (2012)

19. Demirci, K, Dirik, F: Four-dimensional matrix transformation and rate of A-statistical convergence of periodic functions. Math. Comput. Model. 52, 1858-1866 (2010)

20. Mursaleen, $M$, Alotaibi, A: Korovkin type approximation theorem for functions of two variables through statistical A-summability. Adv. Differ. Equ. 2012, Article ID 65 (2012). doi:10.1186/1687-1847-2012-65

21. Mursaleen, M, Kiliçman, A: Korovkin second theorem via B-statistical A-summability. Abstr. Appl. Anal. 2013, Article ID 598963 (2013). doi:10.1155/2013/598963

22. Aral, A, Doğru, O: Bleimann, Butzer and Hahn operators based on q-integers. J. Inequal. Appl. 2007, Article ID 79410 (2007). doi:10.1155/2007/79410

23. Aral, A, Gupta, V: On q-analogue of Stancu-Beta operators. Appl. Math. Lett. 25, 67-71 (2012) 
24. Mursaleen, M, Khan, A: Statistical approximation properties of modified $q$-Stancu-Beta operators. Bull. Malays. Math. Soc., 36(3), 683-690 (2013)

25. Ersan, S, Doğru, O: Statistical approximation properties of $q$-Bleimann, Butzer and Hahn operators. Math. Comput. Model. 49, 1595-1606 (2009)

26. Gupta, V, Radu, C: Statistical approximation properties of $q$-Baskokov-Kantorovich operators. Cent. Eur. J. Math. 7(4), 809-818 (2009)

27. Örkcü, M, Doğru, O: Weighted statistical approximation by Kantorovich type q-Szász-Mirakjan operators. Appl. Math. Comput. 217, 7913-7919 (2011)

28. Radu, C: Statistical approximation properties of Kantorovich operators based on $q$-integers. Creative Math. Inform. 17(2), 75-84 (2008)

29. Mursaleen, M, Khan, A, Srivastava, HM, Nisar, KS: Operators constructed by means of $q$-Lagrange polynomials and A-statistical approximation. Appl. Math. Comput. 219, 6911-6918 (2013)

30. Alotaibi, A, Mursaleen, M: A-Statistical summability of Fourier series and Walsh-Fourier series. Appl. Math. Inform. Sci. 6(3), 535-538 (2012)

31. Erdélyi, A, Magnus, W, Berhettinger, F, Tricomi, FG: Higher Transcendental Functions, vol. III. McGraw-Hill, New York (1953)

32. Chan, WC-C, Chyan, C-J, Srivastava, HM: The Lagrange polynomials of several variables. Integral Transforms Spec. Funct. 12, 139-148 (2001)

33. Chen, KY, Liu, SJ, Srivastava, HM: Some new results for the Lagrange polynomials in several variables. ANZIAM J. 49, 243-258 (2007)

34. Altin, A, Erkuş, E: On a multivariable extension of the Lagrange-Hermite polynomials. Integral Transforms Spec. Funct. 17, 239-244 (2006)

35. Erkuş, E, Srivastava, HM: A unified presentation of some families of multivariable polynomials. Integral Transforms Spec. Funct. 17, 267-273 (2006)

36. Liu, S-J, Lin, S-D, Srivastava, HM, Wong, M-M: Bilateral generating functions for the Erkus-Srivastava polynomials and the generalized lauricella functions. Appl. Math. Comput. 218, 7685-7693 (2012)

10.1186/1029-242X-2013-585

Cite this article as: Mursaleen et al.: Some approximation results for generalized Kantorovich-type operators. Journal of Inequalities and Applications 2013, 2013:585

\section{Submit your manuscript to a SpringerOpen ${ }^{\circ}$ journal and benefit from:}

- Convenient online submission

- Rigorous peer review

- Immediate publication on acceptance

Open access: articles freely available online

- High visibility within the field

- Retaining the copyright to your article 